\title{
Measurement of Patient Confidence in Self-Management of Atrial Fibrillation: Initial Validation of The Confidence in Atrial fibriLlation Management (CALM) Scale
}

\author{
Connor Tripp ${ }^{1}$, Anil Gehi ${ }^{2}$, Lindsey Rosman ${ }^{3}$, Scarlett Anthony ${ }^{1}$, and Samuel Sears ${ }^{1}$ \\ ${ }^{1}$ East Carolina University \\ ${ }^{2}$ University of North Carolina Chapel Hill \\ ${ }^{3}$ University of North Carolina at Chapel Hill School of Medicine
}

December 27, 2020

\begin{abstract}
Abstract Background: The patient experience of atrial fibrillation (AF) involves several daily self-care behaviors and ongoing confidence to manage their condition. Currently, no standardized self-report measure of AF patient confidence exists. The purpose of this study is to establish the reliability and validity of a newly developed confidence in AF management measure. Methods: This study provides preliminary analysis of the Confidence in Atrial FibriLlation Management (CALM) scale, which was rationally developed to measure patient confidence related to self-management of AF. The scale was provided to a sample of AF patients $\mathrm{N}=120$, (59\% male) electronically through a patient education platform. Principle components analysis (PCA) and Cronbach's alpha were employed to provide preliminary assessment of the validity and reliability of the measure. Results: PCA identified a four-factor solution. Internal consistency of the CALM was considered excellent with Cronbach's $\alpha=.910$. Additional PCA confirmed the value of a single factor solution to produce a total confidence score for improved utility and ease of clinical interpretation. Conclusions: Initial assessment of a novel scale measuring patient confidence in managing AF provided promising reliability and validity. Patient confidence in self-management of AF may prove useful as a key marker and endpoint of the patient experience beyond QOL.
\end{abstract}

Measurement of Patient Confidence in Self-Management of Atrial Fibrillation:

Initial Validation of The $\mathbf{C}$ onfidence in $\mathbf{A}$ trial fibriL lation $\mathbf{M}$ anagement (CALM) Scale

Connor Tripp, M.S. ${ }^{1}$, Anil K. Gehi, M.D. ${ }^{2}$, Lindsey Rosman, Ph.D. ${ }^{2}$,

Scarlett Anthony, B.A. ${ }^{1}, \&$ Samuel F. Sears, Ph.D. ${ }^{1}$

${ }^{1}$ Department of Psychology, East Carolina University, Greenville, North Carolina

${ }^{2}$ Department of Medicine, Division of Cardiology, University of North Carolina at Chapel Hill, Chapel Hill, $\mathrm{NC}$

${ }^{3}$ Department of Cardiovascular Sciences, Brody School of Medicine, East Carolina University, Greenville, North Carolina

\section{Correspondence:}

Samuel F. Sears, PhD

East Carolina University

Department of Psychology 
104 Rawl Bldg

Greenville, NC 27858

searss@ecu.edu

Word Count:

Keywords: atrial fibrillation, self-care, health behaviors

\section{Conflict of Interest Disclosures:}

Samuel F. Sears, PhD: Honoraria/Consulting Fees: Medtronic, Abbott, Milestone Pharmaceutical, Zoll Medical; Research Grants: Medtronic, Zoll Medical. All research funds are directed to East Carolina University. Anil Gehi, MD: Research Grant: Bristol Myers Squib Foundation, Honoraria/Consulting: Biotronik, Zoll Medical, Abbott. All other authors do not report any potential conflicts of interest

\section{Funding Sources:}

Dr. Rosman's effort was sponsored by a grant from the National Heart, Lung, and Blood Institute (K23HL141644).

\section{Abstract}

Background: The patient experience of atrial fibrillation (AF) involves several daily self-care behaviors and ongoing confidence to manage their condition. Currently, no standardized self-report measure of AF patient confidence exists. The purpose of this study is to establish the reliability and validity of a newly developed confidence in AF management measure.

Methods: This study provides preliminary analysis of the $\mathbf{C}$ onfidence in $\mathbf{A}$ trial FibriL lation $\mathbf{M}$ anagement (CALM) scale, which was rationally developed to measure patient confidence related to self-management of AF. The scale was provided to a sample of AF patients $\mathrm{N}=120$, (59\% male) electronically through a patient education platform. Principle components analysis (PCA) and Cronbach's alpha were employed to provide preliminary assessment of the validity and reliability of the measure.

Results: PCA identified a four-factor solution. Internal consistency of the CALM was considered excellent with Cronbach's $\alpha=.910$. Additional PCA confirmed the value of a single factor solution to produce a total confidence score for improved utility and ease of clinical interpretation.

Conclusions: Initial assessment of a novel scale measuring patient confidence in managing AF provided promising reliability and validity. Patient confidence in self-management of AF may prove useful as a key marker and endpoint of the patient experience beyond QOL.

\section{Introduction}

Atrial fibrillation $(\mathrm{AF})$ is the most common dysrhythmia, affecting 3.5 million people worldwide, and is associated with an increased risk for stroke. ${ }^{1} \mathrm{AF}$ is a progressive condition that involves medical management such as rate control, rhythm control, and anti-coagulation, as well as lifestyle change and self-management behaviors. ${ }^{2,3} \mathrm{AF}$ is also associated with significant psychologic distress, particularly for patients who are symptomatic, perceive AF to be dangerous, or have low AF health literacy ${ }^{4}$; research to date suggests high rates of anxiety and depression, $35 \%$ and $20 \%$, respectively, in AF samples. ${ }^{5}$ Daily management of $\mathrm{AF}$ involves adherence to medical therapies (e.g rate/rhythm control, stroke prophylaxis), management of comorbidities, abstinence from smoking, avoiding alcohol consumption, and sustaining regular physical activity. ${ }^{6}$ Collectively, the psychological and behavioral aspects of coping with and managing AF contribute to the key outcomes of symptom reduction and quality of life..$^{7,8,9,10,11}$

Current research approaches have generally relied on quality of life measures to capture the entirety of the patient experience. For example, the Atrial Fibrillation Effect on Quality-of-life (AFEQT) questionnaire was developed to explicitly measure patients' perceptions of their symptoms, functional impairment, treatment 
concerns, and satisfaction with treatment. ${ }^{12}$ The reliance on QOL measures to capture the totality of the patient experience focuses on the desired endpoint(s), but may overlook possible process and intervention targets such as: self-assessment, self-care maintenance, management, and confidence that have demonstrated value in heart failure treatment. ${ }^{13,14}$ The development of a patient confidence measure for AF patients may broaden the lens for examination of the patient experience and provide additional understanding and targets for intervention. The purpose of this study was to test the reliability and validity of a stand-alone measure of confidence in self-management of $\mathrm{AF}$, the $\mathbf{C}$ onfidence in $\mathbf{A}$ trial FibriL lation Management (CALM) scale.

\section{Methods}

This study was part of a larger project assessing the utility of a web-based self-care intervention for AF patients, within the context of the COVD-19 pandemic. Patients were recruited from the patient panel of electrophysiologists at the University of North Carolina through electronic outreach. Patients who consented and were enrolled in the intervention study completed several electronic surveys prior to and following the intervention, including surveys of demographic information, health information, and knowledge/attitudes/beliefs about COVID-19, as well as other validated surveys.

For the purposes of this study, patient responses on the CALM, PROMIS-29, and AFEQT were analyzed. The PROMIS-29 is a widely validated and used assessment of general quality of life including eight domains: pain intensity and interference, fatigue, sleep disturbance, physical functioning, depression, anxiety, and ability to participate in social roles and activities. ${ }^{15}$ The AFEQT is a well-established and widely used, four-factored descriptive measure of disease-specific patient quality of life, with demonstrated validity and reliability (internal consistency $>.88$ on all factors). ${ }^{12}$ This measure is used to describe the patient experience of AF, specifically symptoms, daily activities, and perceptions of treatment (i.e. concerns and satisfaction). Medical record review was conducted to obtain information about health and relevant comorbidities. This study was approved by the Institutional Review Board at the University of North Carolina at Chapel Hill (IRB \#20-1121).

Items for the CALM measure were patterned after items from the Self-care of Heart Failure Index (SCHFI) (REF: Riegel) and modified by a cardiac electrophysiologist (AG) and clinical health psychologist (SFS) to tailor them to AF. Next, multiple AF providers including medicine, psychology, and nursing provided feedback on relevance and comprehensiveness of the items to reflect the patient experience with confidence and AF. The preliminary items were then employed in the current study.

Statistical Analyses. The current study utilized survey responses, prior to intervention, in order to establish the validity of a stand-alone measure of confidence in self-management of AF, the CALM, and to describe and compare scores on this measure with other survey data (e.g. AFEQT).

Principle Components Analysis (PCA) was used to analyze the 16-item CALM measure, in order to group variables into key components. A varimax rotation was employed to simplify each component, minimizing the amount of variance within each component and maximizing the variance between components. Cronbach's alpha was then used to assess reliability of the total measure, as well as the reliability of the individual components.

Correlational analyses and independent samples t-tests were then used to assess the relationships and differences in scores on several demographic, health, and QOL variables.

\section{Results}

Demographic and self-reported AF-related information, including relevant comorbidities, reports on health behavior, psychologic diagnoses, PROMIS scores, and AFEQT scores for this sample is provided in Table 1.

\section{Demographics}

Age and BMI

Age
$\mathbf{m}(\mathrm{SD})$; range

71.83 years $( \pm 11.14) ; 24-97$ years
$\mathbf{m}(\mathrm{SD})$; range

71.83 years $( \pm 11.14) ; 24-97$ years 


\begin{tabular}{|c|c|c|}
\hline \multicolumn{3}{|l|}{ Demographics } \\
\hline$\overline{\mathrm{BMI}}$ & $29( \pm 8.06)$ & $29( \pm 8.06)$ \\
\hline Gender & $\mathrm{n}(\%)$ & n (\%) \\
\hline Men & $71(59 \%)$ & $71(59 \%)$ \\
\hline Women & $49(41 \%)$ & $49(41 \%)$ \\
\hline Marital Status & n $(\%)$ & n $(\%)$ \\
\hline Married or Partnered & $93(78 \%)$ & $93(78 \%)$ \\
\hline Single & $14(12 \%)$ & $14(12 \%)$ \\
\hline Widowed & $8(7 \%)$ & $8(7 \%)$ \\
\hline Divorced & $6(5 \%)$ & $6(5 \%)$ \\
\hline Race & n (\%) & n (\%) \\
\hline White/Caucasian & $116(97 \%)$ & $116(97 \%)$ \\
\hline Black/African American & $1(1 \%)$ & $1(1 \%)$ \\
\hline Hispanic & $2(2 \%)$ & $2(2 \%)$ \\
\hline Other & $1(1 \%)$ & $1(1 \%)$ \\
\hline Occupational Status & n (\%) & n (\%) \\
\hline Employed & $25(21 \%)$ & $25(21 \%)$ \\
\hline Retired or Unemployed & $77(64 \%)$ & $77(64 \%)$ \\
\hline Unknown & $18(15 \%)$ & $18(15 \%)$ \\
\hline \multicolumn{3}{|l|}{ Medical Information } \\
\hline AF Type & n (\%) & n (\%) \\
\hline Paroxysmal & $90(75 \%)$ & $90(75 \%)$ \\
\hline Persistent or Permanent & $28(23 \%)$ & $28(23 \%)$ \\
\hline Unsure & $1(1 \%)$ & $1(1 \%)$ \\
\hline History of Surgical Intervention for AF & n (\%) & $\mathrm{n}(\%)$ \\
\hline Ablation & $47(39 \%)$ & $47(39 \%)$ \\
\hline Left Atrial Appendage Occlusion & $5(4 \%)$ & $5(4 \%)$ \\
\hline Cardioversion & $53(44 \%)$ & $53(44 \%)$ \\
\hline Medication Management of AF & n $(\%)$ & n $(\%)$ \\
\hline Anticoagulant & $93(78 \%)$ & $93(78 \%)$ \\
\hline Beta-Blocker & $79(66 \%)$ & $79(66 \%)$ \\
\hline Calcium Channel Blocker & $12(10 \%)$ & $12(10 \%)$ \\
\hline Anti-arrhythmic & $49(41 \%)$ & $49(41 \%)$ \\
\hline $\mathrm{CHA}_{2} \mathrm{DS}_{2}$ VASc Scores & $\mathrm{n}(\%)$ & $\mathrm{n}(\%)$ \\
\hline 0 & $4(3 \%)$ & $4(3 \%)$ \\
\hline 1 & $21(18 \%)$ & $21(18 \%)$ \\
\hline $2+$ & $95(79 \%)$ & $95(79 \%)$ \\
\hline Comorbidities & $\mathrm{n}(\%)$ & $\mathrm{n}(\%)$ \\
\hline Hypertension & $67(56 \%)$ & $67(56 \%)$ \\
\hline Obstructive Sleep Apnea & $45(38 \%)$ & $45(38 \%)$ \\
\hline Coronary Artery Disease & $34(28 \%)$ & $34(28 \%)$ \\
\hline Congestive Heart Failure & $30(25 \%)$ & $30(25 \%)$ \\
\hline Chronic Lung Disease & $29(24 \%)$ & $29(24 \%)$ \\
\hline Prior Myocardial Infarction & $16(13 \%)$ & $16(13 \%)$ \\
\hline Stroke (TIA/CVA) & $16(13 \%)$ & $16(13 \%)$ \\
\hline Diabetes & $14(12 \%)$ & $14(12 \%)$ \\
\hline Chronic Kidney Disease & $13(10.8 \%)$ & $13(10.8 \%)$ \\
\hline \multicolumn{3}{|l|}{ Psychologic and Behavioral Information } \\
\hline Alcohol Use & $\mathrm{n}(\%)$ & n (\%) \\
\hline Mild (1 or less drinks per week) & $26(34 \%)$ & $26(34 \%)$ \\
\hline Moderate ( 7 or less drinks per week) & $33(43 \%)$ & $33(43 \%)$ \\
\hline
\end{tabular}




\begin{tabular}{lll}
\hline Demographics & & \\
\hline Heavy (8+ drinks per week) & $16(21 \%)$ & $16(21 \%)$ \\
Tobacco Use & $\mathbf{n}(\mathbf{\%})$ & $\mathbf{n}(\mathbf{\%})$ \\
Current & $2(2 \%)$ & $2(2 \%)$ \\
Prior & $54(45 \%)$ & $54(45 \%)$ \\
Never & $64(53 \%)$ & $64(53 \%)$ \\
AFEQT Scores & $\mathbf{m}(\mathbf{S D})$ & $\mathbf{m}(\mathbf{S D})$ \\
AFEQT Score & $77.43( \pm 17.46)$ & $77.43( \pm 17.46)$ \\
Psychiatric Diagnoses (per chart review) & $\mathbf{n}(\mathbf{\%})$ & $\mathbf{n}(\mathbf{\%})$ \\
Major Depressive Disorder (MDD) & $16(13 \%)$ & $16(13 \%)$ \\
Generalized Anxiety Disorder (GAD) & $18(15 \%)$ & $18(15 \%)$ \\
PROMIS Measure Scores & & \\
Domain & mean raw score rounded (SD) & $\mathbf{T}$ score \\
Physical Function & $17(3.6)$ & 43.4 \\
Fatigue & $9(4.0)$ & 51.0 \\
Pain Interference & $6(2.8)$ & 52.0 \\
Depressive Symptoms & $6(2.9)$ & 51.8 \\
Anxiety Symptoms & $7(3.3)$ & 53.7 \\
Social Roles \& Activities & $15(4.4)$ & 50.0 \\
Sleep Disturbance & $9(3.6)$ & 48.4 \\
\hline
\end{tabular}

\section{CALM Validity and Reliability}

Initial Factor Analysis. The CALM measure contains 16-items related to various aspects of care, asking patients to indicate the level of confidence they feel in each aspect (i.e. no confidence, somewhat confident, very confident, extremely confident). These 16 items were entered into a PCA with varimax rotation and yielded four components; analysis yielded $68 \%$ of variance in items was explained by these four components (Table 2). The factor loadings were relatively free of multiple loadings and no items warranted deletion.

Table 2. CALM Components

External Support (In general,

how confident are you that...) $\quad$ Factor Loadings Sub Scale $\quad$ Factor Loadings Full Scale

Your doctor understands your

.799

.581

AFib condition?

You can get the emotional

support you need to manage your

AFib?

You can get the practical support

you need to manage your AFib?

You can follow the treatment

.731

.790

advice you have been given?

You can reach your doctor or

.696

nurse for guidance?

.662

.614

Awareness of Change (In

general, how confident are you

that..)

You can get the information you

need about your AFib condition?

You can evaluate the severity of

.799

.716 your symptoms? 


\begin{tabular}{lll}
\hline $\begin{array}{l}\text { External Support (In general, } \\
\text { how confident are you that...) }\end{array}$ & Factor Loadings Sub Scale & Factor Loadings Full Scale \\
\hline $\begin{array}{l}\text { You can recognize changes in your } \\
\text { health if they occur? }\end{array}$ & .836 & .699 \\
$\begin{array}{l}\text { You know when to seek } \\
\text { emergency medical attention? }\end{array}$ & .682 & .702 \\
$\begin{array}{l}\text { Self-Care and Relief (In } \\
\text { general, how confident are you } \\
\text { that...) }\end{array}$ & & \\
$\begin{array}{l}\text { You can keep yourself free of } \\
\text { AFib symptoms? }\end{array}$ & .783 & .567 \\
$\begin{array}{l}\text { You are doing what you can to } \\
\text { manage your AFib? }\end{array}$ & .493 & .716 \\
$\begin{array}{l}\text { You can do something to relieve } \\
\text { your symptoms of AFib? }\end{array}$ & .813 & .656 \\
$\begin{array}{l}\text { You can evaluate if a remedy } \\
\text { works for you? }\end{array}$ & .607 & .671 \\
$\begin{array}{l}\text { Safety (In general, how } \\
\text { confident are you that...) }\end{array}$ & & \\
$\begin{array}{l}\text { You will not die during an episode } \\
\text { of AFib? }\end{array}$ & .678 & .600 \\
$\begin{array}{l}\text { You are not having a heart attack } \\
\text { during an episode of AFib? }\end{array}$ & .750 & .646 \\
$\begin{array}{l}\text { You will not have a stroke during } \\
\text { an episode of AFib? }\end{array}$ & .855 & .428 \\
\hline
\end{tabular}

Higher Order Factor Analysis. These 16 items were then entered into a PCA with a predetermined number of components to extract, 1 , in order to determine if total scores could be used and interpretable; results suggested that a total score was supported, with each item producing correlations between .428 and .828 (Table 3). Internal consistency of the CALM was considered excellent with Cronbach's $\alpha=.910$. Totals and subscale scores by section are presented in Table 3 .

Table 3. CALM Scores

\begin{tabular}{llllll}
\hline Measure & Highest Possible & Lowest Possible & m (SD) & Highest Score in Sample & Lowe \\
\hline CALM: Total Confidence & 48 & 0 & $29.1(8.7)$ & 48 & 9 \\
CALM: External Support & 15 & 0 & $10.7(2.9)$ & 15 & 4 \\
CALM: Awareness of Change & 12 & 0 & $7.5(2.9)$ & 12 & 1 \\
CALM: Self-Care and Relief & 12 & 0 & $5.7(2.7)$ & 12 & 0 \\
CALM: Safety & 9 & 0 & $5.2(2.1)$ & 9 & 1 \\
\hline
\end{tabular}

Comparisons of Demographic and Health Variables. Pearson correlation analysis did not indicate a significant relationship between age and confidence in self-management of $\mathrm{AF}(p=.468)$ or BMI and confidence and AF self-management $(p=.5)$.

Independent samples t-tests were used to assess differences in CAFS scores between different demographic, health, and AF specific variables. Interestingly, results indicated no significant differences between mean scores for women $(\mathrm{m}=29.7, \mathrm{SD}=8.8)$ and men $(\mathrm{m}=28.7, \mathrm{SD}=8.7), t(100.394)=-.608, p=.545$. No significant differences were detected between those with $\mathrm{CHA}_{2} \mathrm{DS}_{2} \mathrm{VASc}$ scores of two or more and those with one or less $t(48.1)=-.602, p=.550$. There were also no significant differences between those with 
past diagnoses of MDD $(\mathrm{m}=27.5, \mathrm{SD}=8.4)$ and those without $(\mathrm{m}=29.3, \mathrm{SD}=8.8), t(15.7)=.743, p$ $=.468$. However, there was a significant difference between scores for those with past diagnoses of GAD $(\mathrm{m}$ $=25.3, \mathrm{SD}=6.6)$ and those without $(\mathrm{m}=29, \mathrm{SD}=9.0), t(31.2)=2.49, p=.018$, likely indicating that those with anxiety have less confidence in self-management of AF than those without anxiety.

Related to procedural treatment of $\mathrm{AF}$, an independent samples t-test suggested no significant difference in confidence scores between those patients who had undergone an ablation for AF management $(\mathrm{m}=30.2$, SD $=7.0)$ and those who had not $(\mathrm{m}=28.7, \mathrm{SD}=9), t(103.3)=-.945, p=.347$. There was also no significant difference in confidence scores between those who had undergone an LAA occlusion $(\mathrm{m}=26.2, \mathrm{SD}=3.1)$ than those who had not $(\mathrm{m}=29.5, \mathrm{SD}=8.7), t(7.59)=1.98, p=.085$. Interestingly, there was a significant difference in confidence scores between those who had undergone a cardioversion for $\mathrm{AF}(\mathrm{m}=32$, SD $=$ $7.2)$ and those who had not $(\mathrm{m}=27.4, \mathrm{SD}=9.0), \mathrm{t}(104.4)=-.297, \mathrm{p}=.004)$, suggesting those who had undergone cardioversion had significantly more confidence in self-management of AF.

Related to medication for AF management, independent samples t-tests indicated that there were no significant differences between those patients prescribed anticoagulants $(\mathrm{m}=29.8, \mathrm{SD}=8.6)$ and those that were not $(\mathrm{m}=26.6, \mathrm{SD}=8.9), t(36.0)=-1.58, p=.123$, or between those who were prescribed beta-blockers $(\mathrm{m}=29.4, \mathrm{SD}=9.0)$ and those that were not $(\mathrm{m}=28.2, \mathrm{SD}=8.6), \mathrm{t}(63.6)=-.659, \mathrm{p}=.513$. Interestingly, there was a significant difference between those who were prescribed anti-arrhythmic medications $(\mathrm{m}=32.7$, $\mathrm{SD}=7.9)$ and those that were not $(\mathrm{m}=26.7, \mathrm{SD}=8.6), \mathrm{t}(97.0)=-3.767, \mathrm{p}<.001$, suggesting that individuals prescribed anti-arrhythmic medications for AF had greater confidence in self-management than individuals without anti-arrhythmic medications.

Comparison with Quality of Life . Lastly, a simple linear regression analysis was used to assess the ability of the CALM total scores to predict AF quality of life using AFEQT total scores (Figure 1). Results of this analysis suggested that CALM total scores predicted increased AFEQT total scores $\left(r^{2}=.142, p<.001\right)$. In other words, greater confidence in AF self-management predicted better quality of life with AF, with $14.2 \%$ of variance in quality of life being explained by confidence in AF self-management.

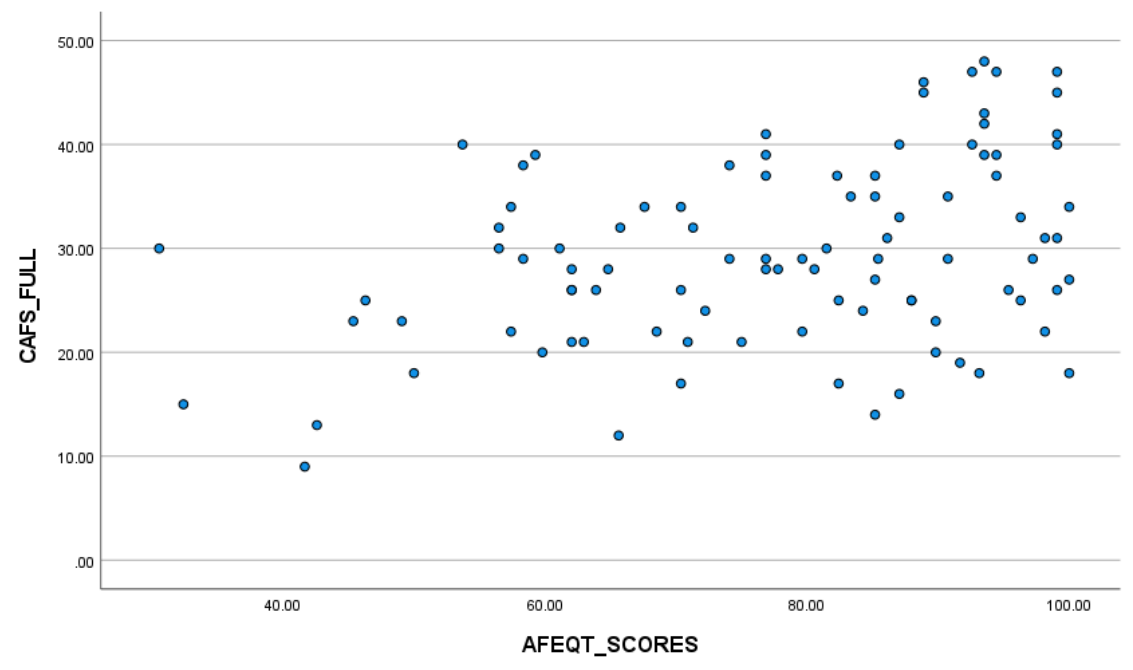

\section{Discussion}

The current study examined a new scale for measuring patient confidence in self-management of AF. Initial reliability and validity metrics were promising and suggest potential utility though further validation testing is warranted. The CALM measure containing 16 self-report items underwent principle component analysis and a four-factor solution was identified. The major aspects included scales of external support, awareness of change, self-care and relief, and safety. Additional analyses indicated that a total confidence score could 
be derived and was psychometrically sound. Confidence in AF was similar between men and women, but significantly different between patients with and without a documented anxiety disorder, those with the experience of cardioversion, and those who have taken anti-arrhythmic medication.

These data suggest that background experiential variables may exert some influence on perceptions of AF and QOL. Previous studies have linked the experience of cardioversion with better QOL reports and the presence of anxiety to worse QOL. ${ }^{16,17}$ The utility of the CALM was further established, as total confidence was a significant predictor of AF QOL, but only accounted for $14 \%$ of the variance. Therefore, this initial work indicates that the CALM provides a brief, easy-to-use in-clinic measure of patient confidence in AF self-management with some promising validity that should be used in conjunction with QOL measures. With additional validation and testing, AF confidence may serve as a target for clinical interventions and ongoing clinical care. Confidence in AF self-management could provide a process measure for achieving successful self-care and better QOL.

Patient confidence or self-efficacy in managing chronic disease has been well-examined and generally confidence tends to diminish as the illness burden increases. ${ }^{18}$ This finding is particularly relevant as patients with AF are likely to have multiple co-morbidities, multiple treatment modalities, and multiple risk factors to address suggesting a high degree of management burden. ${ }^{11}$ Previous examination of confidence in AF self-management was completed by employing an online survey of women with $\mathrm{AF} .{ }^{10}$ Results indicated that the most salient item of the survey emphasized, "taking active role in my health is important in how I feel." Confidence in cardiovascular conditions has not been as well-studied but when the construct of confidence was studied, researchers generally focused on confidence in one target behavior. ${ }^{9}$

The awareness and measurement of patient attitudes and patient confidence represents a new avenue for cardiac electrophysiology. The history of cardiac electrophysiology has primarily focused on technologic advances and procedural based improvements. The emergence of data related to the potential value of risk factor management in AF has prompted increased attention to multi-disciplinary care to improve outcomes regardless of treatment strategy. ${ }^{19}$ The identification of cardiac fitness, obesity, alcohol use, sleep apnea, and other risk factors indicates that patients increasingly must be equal partners with cardiac electrophysiology teams. ${ }^{20}$ Psychological confidence represents a necessary, but not sufficient, component of achieving better health outcomes. The introduction of a brief, clinic-ready AF confidence measure (i.e. CALM measure) could provide information about how patients perceive their capability and potentially how patient education, and behavioral intervention are likely to be effective. In short, patients who do not believe that they are capable of acquiring sufficient social and informational support, making changes, engaging in effective selfcare and feeling safe again to return to physical activities are probably less likely to benefit without targeted intervention. Future research must examine this notion empirically, but it seems plausible.

Despite the promise the CALM holds for understanding patient confidence in self-management of AF, the study has some limitations. First, it should be noted that the sample was mostly white (97\%) with only one Hispanic and two African American participants. Convenience sampling was solely utilized in this round of testing of the CALM. Future studies using the CALM should focus on more diverse samples in order to test the generalizability of the findings. Secondly, the study was conducted during COVID-19, therefore we cannot entirely exclude unknown potential confounding effects of the pandemic. Patient experience and self-care behaviors may not accurately represent this population's reality under non-pandemic conditions. Social support may be limited and may have affected that set of items on the measure. Thus, continued research using the CALM to understand patient confidence in self-management of AF is needed considering the complexity of self-care, clinical management, and understanding of AF. Further research is needed to establish clinically meaningful cut-off scores to indicate the need for intervention.

The current study establishes the reliability and validity of a new patient reported outcome measure of confidence in atrial fibrillation, The $\mathbf{C}$ onfidence in $\mathbf{A}$ trial FibriL lation $\mathbf{M}$ anagement (CALM) scale, providing a total score and subscales of confidence. Additional analyses indicate that AF confidence is a significant predictor of AF QOL. As multidisciplinary care for AF continues to mature, greater attention and intervention on the patient experience and psychological functioning will likely become important. Preliminary data 
suggests the new measure, CALM, will benefit from additional psychometric testing. We seek to make the measure widely available for use at no charge for future research as we hope it becomes a useful addition to understanding the patient experience alongside AF QOL.

References

1. Wang X, Fu Q, Song F, Li W, Yin X, Yue W, et al. Data on prevalence of atrial fibrillation and its association with stroke in low-, middle-, and high-income regions of china. Data in Brief. 2018;19:18221827.

2. Chung MK, Refaat M, Shen WK, Kutyifa V, Cha YM, Di Biase L, Baranchuk A, Lampert R, Natale A, Fisher J, Lakkireddy DR; ACC Electrophysiology Section Leadership Council. Atrial Fibrillation: JACC Council Perspectives. J Am Coll Cardiol. 2020 Apr 14;75(14):1689-1713. doi: 10.1016/j.jacc.2020.02.025. PMID: 32273035.

3. Chung MK, Eckhardt LL, Chen LY, Ahmed HM, Gopinathannair R, Joglar JA, et al. Lifestyle and risk factor modification for reduction of atrial fibrillation: A scientific statement from the American Heart Association. Circulation. 2020;141(16):e750-72.

4. McCabe, P.J. and Barnason, S.A. Illness perceptions, coping strategies, and symptoms contribute to psychological distress in patients with recurrent symptomatic atrial fibrillation. JCN. 2012;27 (5):431444.

5. Polikandrioti M, Koutelekos I, Vasilopoulos G, Gerogianni G, Gourni M, Zyga S, Panoutsopoulos G. Anxiety and Depression in Patients with Permanent Atrial Fibrillation: Prevalence and Associated Factors. Cardiol Res Pract. 2018 Feb 19;7408129.

6. Di Benedetto L, Michels G, Luben R, Khaw K-T, Pfister R. Individual and combined impact of lifestyle factors on atrial fibrillation in apparently healthy men and women: The EPIC-Norfolk prospective population study. Eur J Prev Cardiol. 2018;25(13):1374-83.

7. Walters TE, Wick K, Tan G, Mearns M, Joseph SA, Morton JB, et al. Symptom severity and quality of life in patients with atrial fibrillation: Psychological function outweighs clinical predictors. Int J Cardiol. 2019;279:84-9.

8. Garimella RS, Sears SF, Gehi AK. Depression and physical inactivity as confounding the effect of obesity on atrial fibrillation. Am J Cardiol. 2016;117(11):1760-4.

9. Chen LY, Chung MK. Risk factor modification: Another win for our fight against atrial fibrillation. EP Europace. 2019;21(4):527-528.

10. Macario E, ScD MS, EdM S, T. Y, PhD MPH, Campbell SM, et al. Quality of life experiences among women with atrial fibrillation: Findings from an online survey. Women's Health Issues. 2016;26(3):288297.

11. Bamgbade BA, Sanghai SR, McManus DD, Lessard D, Waring ME, Forrester S, et al. Psychosocial and cognitive multimorbidity and health-related quality of life and symptom burden in older adults with atrial fibrillation: The systematic assessment of geriatric elements in atrial fibrillation (SAGE-AF) cohort study. Archives of Gerontology and Geriatrics. 2020;90:104117-104117.

12. Spertus J, Dorian P, Bubien R, Lewis S, Godejohn D, Reynolds MR, et al. Development and validation of the atrial fibrillation effect on Quality-of-life (AFEQT) questionnaire in patients with atrial fibrillation. Circulation Arrhythmia and Electrophysiology. 2011;4(1):15-25.

13. Barbaranelli C, Lee CS, Vellone E, Riegel B. Dimensionality and reliability of the self-care of heart failure index scales: Further evidence from confirmatory factor analysis. Research in Nursing \& Health. 2014;37(6):524-537.

14. Riegel B, Barbaranelli C, Carlson B, Sethares KA, Daus M, Moser DK, et al. Psychometric testing of the revised self-care of heart failure index. The Journal of Cardiovascular Nursing. 2019;34(2):183-192.

15. Hays RD, Spritzer KL, Schalet BD, Cella D. Promis(ß)-29 v2.0 profile physical and mental health summary scores. Qual Life Res. 2018;27:1885-1891

16. Ha ACT, Stewart J, Klein G, Roy D, Connolly S, Koren A, et al. Impact of electrical cardioversion on quality of life for patients with symptomatic persistent atrial fibrillation: Is there a treatment expectation effect? The American Heart Journal. 2020;226:152-160. 
17. Son Y, Baek K, Lee SJ, Seo EJ. Health-related quality of life and associated factors in patients with atrial fibrillation: An integrative literature review. International Journal of Environmental Research and Public Health. 2019;16(17):3042.

18. Finney Rutten LJ, Hesse BW, St. Sauver JL, Wilson P, Chawla N, Hartigan DB, et al. Health selfefficacy among populations with multiple chronic conditions: The value of patient-centered communication. Advances in Therapy. 2016;33(8):1440-1451.

19. Hindricks G, Potpara T, Dagres N, Arbelo E, Bax JJ, Blomström-Lundqvist C, et al. 2020 ESC Guidelines for the diagnosis and management of atrial fibrillation developed in collaboration with the European Association of Cardio-Thoracic Surgery (EACTS). Eur Heart J [Internet]. 2020

20. Donnellan E, Wazni O, Kanj M, Cantillon D, Patel D, Trulock K, et al. impact of nonalcoholic fatty liver disease on arrhythmia recurrence following atrial fibrillation ablation. Journal of the American College of Cardiology. 2020;75(11):508-508. 